\title{
Thermoluminescence of Novel Zinc Oxide Nanophosphors Obtained by Glycine-Based Solution Combustion Synthesis
}

\author{
V. R. Orante-Barrón, ${ }^{1}$ F. M. Escobar-Ochoa, ${ }^{1}$ C. Cruz-Vázquez, ${ }^{1}$ and R. Bernal ${ }^{2}$ \\ ${ }^{1}$ Departamento de Investigación en Polímeros y Materiales, Universidad de Sonora, Apartado Postal 130, 83000 Hermosillo, SON, \\ Mexico \\ ${ }^{2}$ Departamento de Investigación en Física, Universidad de Sonora, Apartado Postal 5-088, 83190 Hermosillo, SON, Mexico
}

Correspondence should be addressed to V. R. Orante-Barrón; vorante77@gmail.com

Received 7 November 2014; Accepted 19 December 2014

Academic Editor: Victor M. Castaño

Copyright (c) 2015 V. R. Orante-Barrón et al. This is an open access article distributed under the Creative Commons Attribution License, which permits unrestricted use, distribution, and reproduction in any medium, provided the original work is properly cited.

\begin{abstract}
High-dose thermoluminescence dosimetry properties of novel zinc oxide nanophosphors synthesized by a solution combustion method in a glycine-nitrate process are presented for the very first time in this work. Sintered particles with sizes ranging between $\sim 500 \mathrm{~nm}$ and $\sim 2 \mu \mathrm{m}$ were obtained by annealing the synthesized $\mathrm{ZnO}$ at $900^{\circ} \mathrm{C}$ during $2 \mathrm{~h}$ in air. X-ray diffraction patterns indicate the presence of the $\mathrm{ZnO}$ hexagonal phase, without any remaining nitrate peaks observed. Thermoluminescence glow curves of $\mathrm{ZnO}$ obtained after being exposed to beta radiation consists of two maxima: one located at $\sim 149^{\circ} \mathrm{C}$ and another at $\sim 308^{\circ} \mathrm{C}$, the latter being the dosimetric component of the curve. The integrated TL fading displays an asymptotic behavior for times longer than $16 \mathrm{~h}$ between irradiation and the corresponding TL readout, as well as a linear behaviour of the dose response without saturation in the studied dose interval (from 12.5 up to $400 \mathrm{~Gy}$ ). Such features place synthesized $\mathrm{ZnO}$ as a promising material for high-dose radiation dosimetry applications.
\end{abstract}

\section{Introduction}

A wide variety of luminescent materials used as thermoluminescent dosimeters (TLD) can be applied in several areas such as environmental and clinical dosimetry (low dose dosimetry), as well as applications related with high radiation doses such as nuclear reactors facilities, food sterilization plants, materials testing, and space dosimetry $[1,2]$. In the case of high-dose dosimetry, thermoluminescence (TL) dose response should not exhibit a sublinear behaviour with a saturation trend, nor superlinear response in the range of interest because the real dose absorbed by the dosimeter can be underestimated or overestimated, respectively. Some materials, in particular conventional TL dosimeters, show a remarkable superlinearity at high-dose levels: thus, the number of available materials for high-dose dosimetry is limited $[1,2]$. Nowadays, the search for new materials for high-dose TL dosimetry applications with high saturation limits represents a considerable challenge in the field of materials science. There are a considerable number of reports regarding the TL response of zinc oxide under exposure with different types of radiation sources [3-6].

Moreover, further investigations related to TL dosimetry features of pure and doped $\mathrm{ZnO}$ nanophosphors obtained by different soft chemistry-based methods had been carried out, proposing this oxide as a promising material for high-dose radiation dosimetry applications [7-15].

As an alternative to soft chemistry methods, solution combustion synthesis (SCS) is a technique in which a highly exothermic redox chemical reaction is carried out between a metal nitrate and a fuel, resulting in nanocrystals with composition consistent with the initial reagents. SCS begins with the complexation of the metallic species (oxidizing agent) prior to the combustion with an organic compound (reducing agent) functioning as the fuel. A large variety of technologically important inorganic compounds can be produced by SCS, such as ceramic oxides, aluminates, cromites, ferrites, manganites, and carbides [16].

Recently, Jagannatha Reddy et al. reported nanocrystalline $\mathrm{ZnO}$ powders which have been synthesized by a low 
temperature solution combustion method using oxalyldihydrazide as fuel. Thermoluminescence (TL) glow curves of gamma irradiated $\mathrm{ZnO}$ nanoparticles exhibit a single broad glow peak at $\sim 343^{\circ} \mathrm{C}[14]$.

SCS based on a glycine-nitrate process represents a mechanistic advantage referring to the chemistry of the process in comparison with the combustion using urea or carboxylate azides such as oxalyldihydrazide as the complexant/fuel. The "zwitterionic" character of glycine allows the effective complexation of metals depending on the ionic size. Alkaline and alkaline-earth ions are more effectively complexed by the carboxylic acid group, while many transitions metals are more effectively complexed by the amino group [17].

Thereby, motivated by the antecedents mentioned above, the purpose of this work is to present for the very first time high-dose TL dosimetry properties of novel zinc oxide obtained by a glycine-nitrate solution combustion synthesis.

\section{Experimental}

2.1. Zinc Oxide Synthesis. An initial $0.25 \mathrm{M}$ zinc nitrate hexahydrate $\left(\mathrm{Zn}\left(\mathrm{NO}_{3}\right)_{2} \cdot 6 \mathrm{H}_{2} \mathrm{O}\right.$, ACS grade, Sigma-Aldrich) solution was prepared using deionized water inside a $400 \mathrm{~mL}$ beaker, where $0.42 \mathrm{~g}$ of glycine $\left(\mathrm{C}_{2} \mathrm{H}_{5} \mathrm{NO}_{2}\right.$, ACS grade, Sigma-Aldrich) was added to the metallic nitrate solution to get a fuel-lean elemental stoichiometric coefficient value $\left(\Phi_{e}=1.75\right)$ for the fuel-oxidizer mixture [18]. The resulting solution was placed in a hot plate and stirred magnetically at 360 RPM during 30 min inside a fume hood. After the stirring process, the solution temperature was increased from room temperature up to $200^{\circ} \mathrm{C}$ during $1 \mathrm{~h}$, and when most of the water content of the solution was evaporated, the temperature was increased up to $500^{\circ} \mathrm{C}$ until a green flame was observed. Once the combustion finished, only the inorganic material powder remained at the bottom of the beaker. The solid within the beaker was then cooled to room temperature and the solid was collected and finally crushed with a pestle in an agate mortar. Powder samples obtained by the SCS technique were thermally annealed inside a Thermolyne 1300 furnace in air at $900^{\circ} \mathrm{C}$ during $2 \mathrm{~h}$. After each annealing, the samples were cooled slowly inside the furnace.

2.2. Materials Characterization. X-ray diffraction data were obtained using a Bruker D8 Advance with $\mathrm{Cu}$ Ka radiation $(\lambda=1.542 \AA)$. Scanning electron microscopy (SEM) images were obtained using JEOL JSM-5410LV equipment. A Risø TL/OSL model TL/OSL-DA-20 unit equipped with a ${ }^{90} \mathrm{Sr}$ beta radiation source was used to perform beta irradiations, thermoluminescence (TL) measurements, and the TL dosimetry characterization. Aliquots of $60 \mathrm{mg}$ of sample in stainless steel cups were used for the readouts. All irradiations were accomplished using a $5 \mathrm{~Gy} / \mathrm{min}$ dose rate at room temperature $\left(22^{\circ} \mathrm{C}\right)$. The TL readouts were carried out under $\mathrm{N}_{2}$ atmosphere using a heating rate of $5^{\circ} \mathrm{C} / \mathrm{s}$.

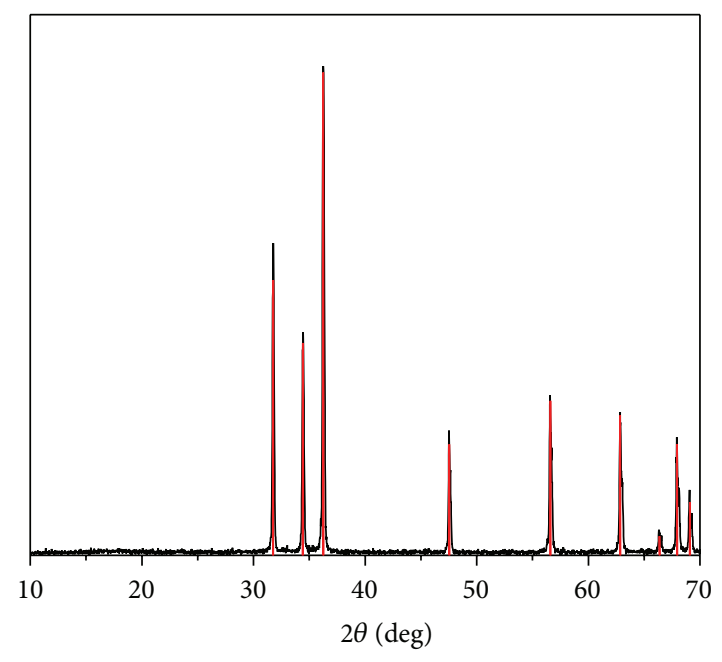

FIGURE 1: X-ray diffraction pattern of annealed samples obtained by SCS. Red vertical lines indicate the diffraction pattern of zinc oxide or zincite (PDF number 036-1251).

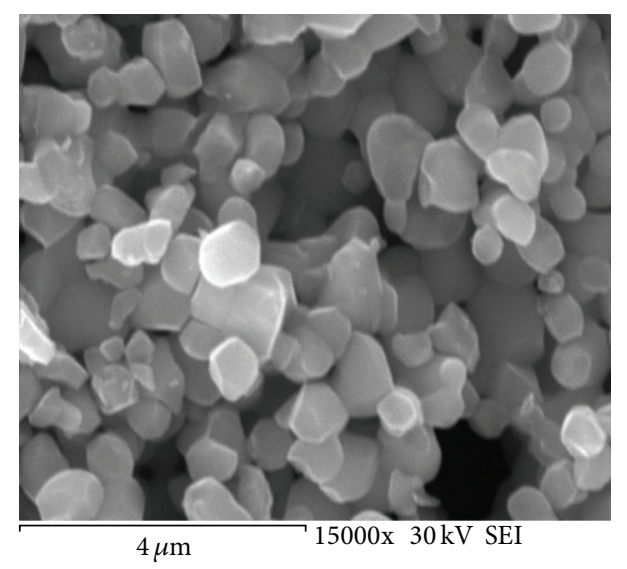

FIGURE 2: SEM image of $\mathrm{ZnO}$ samples synthesized by SCS.

\section{Results and Discussion}

As can be seen in Figure 1, the X-ray diffraction pattern obtained from annealed powder samples obtained by SCS consists of sharp well-defined peaks, which indicates high crystallinity.

Experimental diffraction data correspond to hexagonal zinc oxide or zincite (PDF number 036-1251) represented by red vertical lines, with a remarkable phase purity, since remaining nitrate or organic material diffraction peaks were not observed.

Figure 2 shows the SEM image of $\mathrm{ZnO}$ samples. Sintered particles with sizes ranging between $\sim 500 \mathrm{~nm}$ and $\sim 2 \mu \mathrm{m}$ can be observed, with well-defined particle shapes and no visible porosity.

Thermoluminescence glow curves of $\mathrm{ZnO}$ nanophosphors after being exposed to beta radiation in the dose range from 12.5 up to 400 Gy are shown in Figure 3. Two maxima are observed, one located at $\sim 149^{\circ} \mathrm{C}$ and another at $\sim 308^{\circ} \mathrm{C}$. These maxima shift their positions to lower temperatures 


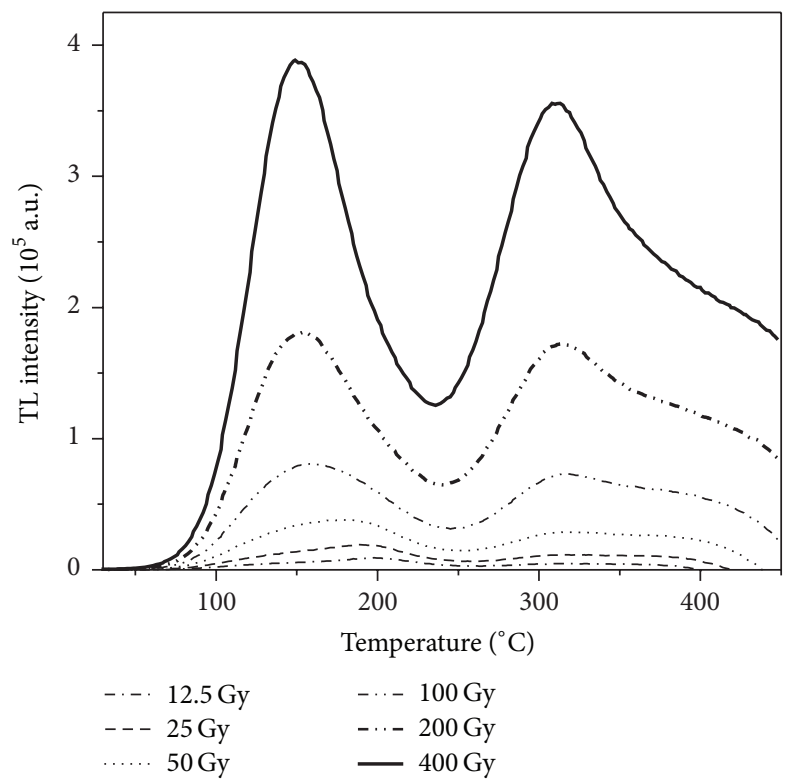

FIGURE 3: Thermoluminescence glow curves of $\mathrm{ZnO}$ nanophosphors obtained by SCS.

as the dose increases, experimental evidence that secondorder kinetics is predominating in the TL processes, although further glow curve deconvolution studies are necessary to better support such assumption [19].

The maximum located at $\sim 308^{\circ} \mathrm{C}$, mainly due to electron recombination from deep trapping states, is expected to be the stable peak since it is located at a temperature higher than $200^{\circ} \mathrm{C}$, whereas the peak located at $\sim 149^{\circ} \mathrm{C}$ can be considered as a low temperature peak and not suitable for TL dosimetry, mainly due to electron recombination from shallow traps [19, 20].

It is worth mentioning that there is no evidence of saturation in the 12.5-200 Gy dose interval for $\mathrm{ZnO} \mathrm{TL}$ glow curves obtained in the present work.

TL glow curves obtained in this work are different compared to the curves of $\mathrm{ZnO}$ synthesized by a combustion process, but using oxalyl dihydrazide $\left(\mathrm{C}_{2} \mathrm{H}_{6} \mathrm{~N}_{4} \mathrm{O}_{2}\right)$ as fuel, reported by Jagannatha Reddy et al. In that work, TL glow curve consists of a single broad peak located at $\sim 343^{\circ} \mathrm{C}$. Also, there is evidence of TL signal saturation as the dose increases in the dose interval studied (10-50 Gy), according to the glow curves shown [14], which is lower than the interval used in the present study.

The integrated TL as a function of the dose, namely, the dose response of $\mathrm{ZnO}$ nanophosphors, is shown in Figure 4. Each point in the plot represents the integrated TL or the area beneath each TL glow curve shown in Figure 3. A linear behavior as well as proportionality in the 12.5-200 Gy dose interval can be observed. There is a sublinear behaviour evidence for doses above $200 \mathrm{~Gy}$. Moreover, a further study is necessary to carry out to determine which is the optimal annealing prior to irradiation in order to eliminate the effect of the peak located at $\sim 149^{\circ} \mathrm{C}$ and, therefore, the sublinear trend for doses above $200 \mathrm{~Gy}$.

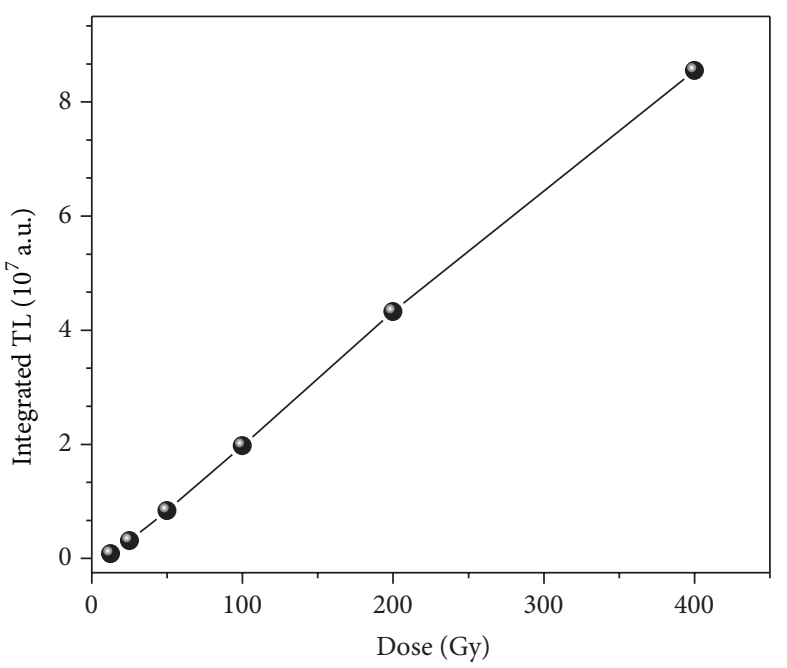

FIGURE 4: Dose response of $\mathrm{ZnO}$ nanophosphors obtained by SCS.

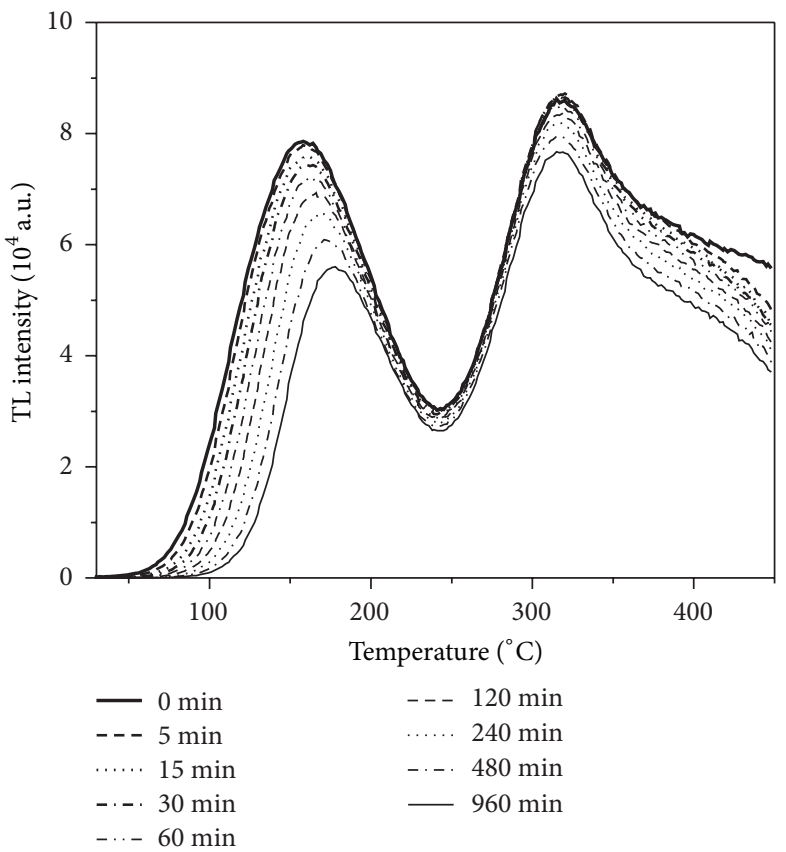

FIgURE 5: TL glow curve evolution of $\mathrm{ZnO}$ nanophosphors, previously exposed to $50 \mathrm{~Gy}$ of beta radiation after each elapsed time between irradiation and the corresponding TL readout (inner text).

Figure 5 shows the TL glow curve evolution obtained previously exposing $\mathrm{ZnO}$ nanophosphors to $50 \mathrm{~Gy}$ of beta radiation, increasing the elapsed time between each irradiation and the corresponding TL readout until $16 \mathrm{~h}$. It can be observed that TL intensity of the component located at $\sim 149^{\circ} \mathrm{C}$ decreases in a higher proportion as time increases, compared to the maximum at $\sim 308^{\circ} \mathrm{C}$ which is a more stable component since it represents recombination of charge carriers such as electrons previously trapped at deeper localized metastable energy states within the band gap of $\mathrm{ZnO}$.

Figure 6 shows the total TL signal as a function of the elapsed time between irradiation and the corresponding TL 


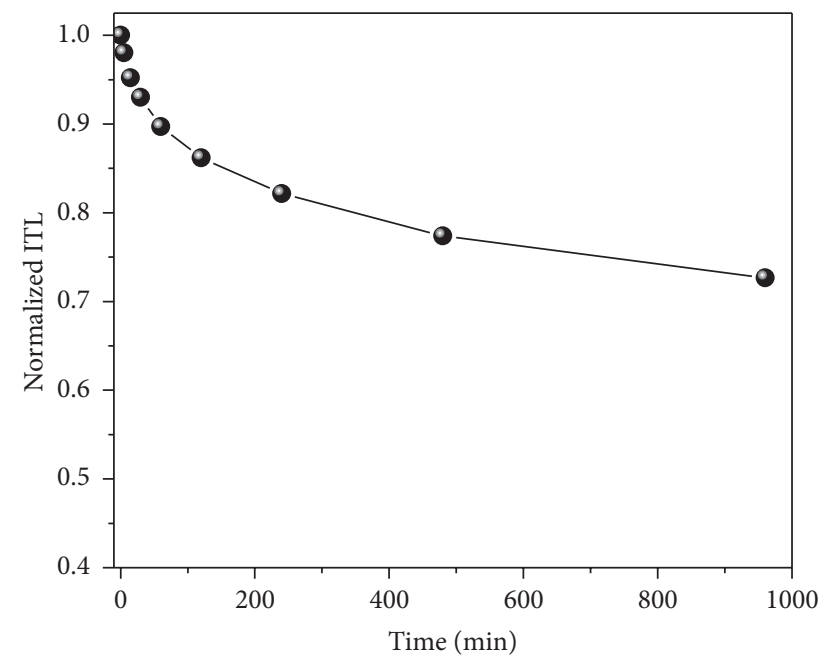

FIGURE 6: Normalized integrated TL as a function of the elapsed time between irradiation and the corresponding TL readout of $\mathrm{ZnO}$ nanophosphors.

readout of $\mathrm{ZnO}$ nanophosphors. Each point in the plot represents the integrated TL or the area beneath each TL glow curve shown in Figure 5. The signal faded down $25 \%$ past $9 \mathrm{~h}$ after irradiation, with an asymptotic behaviour for times longer than $16 \mathrm{~h}$. Such behaviour is mainly due to the $\sim 308^{\circ} \mathrm{C}$ peak which can be suitable for dosimetry applications such as radiotherapy and food irradiation industry.

\section{Conclusions}

It was feasible to synthesize through a glycine-nitrate combustion process novel $\mathrm{ZnO}$ nanophosphors with phase purity and with remarkable TL dosimetry properties under betaparticle irradiation, according to the results obtained in this work. Dose response without saturation evidence and a linear trend for doses up to $200 \mathrm{~Gy}$ as well as the asymptotic behaviour of the TL fading for times longer than $16 \mathrm{~h}$ are experimental evidence for proposing annealed $\mathrm{ZnO}$ as a promising material aimed for high-dose radiation dosimetry applications such as radiotherapy and food irradiation industry.

\section{Conflict of Interests}

The authors declare that there is no conflict of interests regarding the publication of this paper.

\section{Acknowledgment}

The corresponding author gratefully acknowledges the financial support to this work from PROMEP through Grant PROMEP/103.5/11/4462.

\section{References}

[1] R. Chen and S. W. S. McKeever, Theory of Thermoluminescence and Related Phenomena, World Scientific, Singapore, 1997.
[2] S. W. S. McKeever, M. Moscovitch, and P. D. Townsend, Thermoluminescence Dosimetry Materials: Properties and Uses, Nuclear Technology Publishing, Ashford, UK, 1995.

[3] D. de Muer and W. Maenhout-van der Vorst, “Thermoluminescence of ZnO powder," Physica, vol. 39, no. 1, pp. 123-132, 1968.

[4] D. Diwan, S. Bhushan, and S. P. Kathuria, "Thermoluminescence of $\mathrm{ZnO}: \mathrm{Cu}$, La under UV and $\gamma$-ray irradiation," Crystal Research and Technology, vol. 19, no. 9, pp. 1265-1269, 1984.

[5] S. Bhushan, D. Diwan, and S. P. Kathuria, "Thermoluminescence of $\mathrm{ZnO}: \mathrm{Pr}$ and $\mathrm{ZnO}: \mathrm{Gd}$ under UV, $\beta$ - and $\gamma$-ray irradiations," Physica Status Solidi (A): Applied Research, vol. 83, no. 2, pp. 605-611, 1984.

[6] C. Coskun, D. C. Look, G. C. Farlow, and J. R. Sizelove, "Radiation hardness of $\mathrm{ZnO}$ at low temperatures," Semiconductor Science and Technology, vol. 19, no. 6, pp. 752-754, 2004.

[7] C. Cruz-Vázquez, R. Bernal, S. E. Burruel-Ibarra, H. GrijalvaMonteverde, and M. Barboza-Flores, "Thermoluminescence properties of new $\mathrm{ZnO}$ nanophosphors exposed to beta irradiation," Optical Materials, vol. 27, no. 7, pp. 1235-1239, 2005.

[8] C. Cruz-Vázquez, S. E. Burruel-Ibarra, H. Grijalva-Monteverde, V. Chernov, and R. Bernal, "Thermally and optically stimulated luminescence of new $\mathrm{ZnO}$ nanophosphors exposed to beta particle irradiation," Radiation Effects and Defects in Solids, vol. 162, no. 10-11, pp. 737-743, 2007.

[9] U. Pal, R. Meĺndrez, V. Chernov, and M. Barboza-Flores, “Thermoluminescence properties of $\mathrm{ZnO}$ and $\mathrm{ZnO}: \mathrm{Yb}$ nanophosphors," Applied Physics Letters, vol. 89, no. 18, Article ID 183118, 2006.

[10] U. Pal, R. Meléndrez, V. Chernov, and M. Barboza-Flores, "Effect of $\mathrm{Yb}$ doping on the afterglow and thermoluminescent properties of $\mathrm{ZnO}$ nanophosphors," Journal of Nanoscience and Nanotechnology, vol. 8, no. 12, pp. 6513-6518, 2008.

[11] M. Bedir, M. Öztas, A. N. Yazici, and E. V. Kafadar, "Characterization of undoped and $\mathrm{Cu}$-doped $\mathrm{ZnO}$ thin films deposited on glass substrates by spray pyrolysis," Chinese Physics Letters, vol. 23, no. 4, pp. 939-942, 2006.

[12] A. K. Srivastavaa, K. Ninagawab, S. Toyodab, B. R. Chakrabortya, and S. Chandrac, "Induced thermoluminescence of X-ray irradiated nanostructured zinc oxide," Optical Materials, vol. 32, no. 2, pp. 410-413, 2009.

[13] C. Cruz-Vázquez, H. A. Borbón-Nuñez, V. R. Orante-Barrón, S. E. Burruel-Ibarra, V. M. Castaño, and R. Bernal, "Synthesis and thermoluminescence of new $\mathrm{ZnO}$ phosphors," MRS Proceedings, vol. 1278, pp. S8-S33, 2010.

[14] A. Jagannatha Reddy, M. K. Kokila, H. Nagabhushana et al., "EPR, thermo and photoluminescence properties of $\mathrm{ZnO}$ nanopowders," Spectrochimica Acta Part A: Molecular and Biomolecular Spectroscopy, vol. 81, no. 1, pp. 59-63, 2011.

[15] C. Cruz-Vázquez, H. A. Borbón-Nuñez, R. Bernal, J. A. GasparArmenta, and V. M. Castaño, "Thermally stimulated luminescence of Mg-doped ZnO Nanophosphors," Radiation Effects and Defects in Solids, vol. 169, no. 5, pp. 380-387, 2014.

[16] K. C. Patil, S. T. Aruna, and S. Ekambaram, "Combustion synthesis," Current Opinion in Solid State and Materials Science, vol. 2, no. 2, pp. 158-165, 1997.

[17] L. A. Chick, L. R. Pederson, G. D. Maupin, J. L. Bates, L. E. Thomas, and G. J. Exarhos, "Glycine-nitrate combustion synthesis of oxide ceramic powders," Materials Letters, vol. 10, no. 1-2, pp. 6-12, 1990.

[18] L. E. Shea, J. McKittrick, O. A. Lopez, and E. Sluzky, "Synthesis of red-emitting, small particle size luminescent oxides using 
an optimized combustion process," Journal of the American Ceramic Society, vol. 79, no. 12, pp. 3257-3265, 1996.

[19] C. Furetta and P. S. Weng, Operational Thermoluminescence Dosimetry, World Scientific, Singapore, 1998.

[20] S. W. S. McKeever, Thermoluminescence of Solids, Cambridge University Press, New York, NY, USA, 1985. 

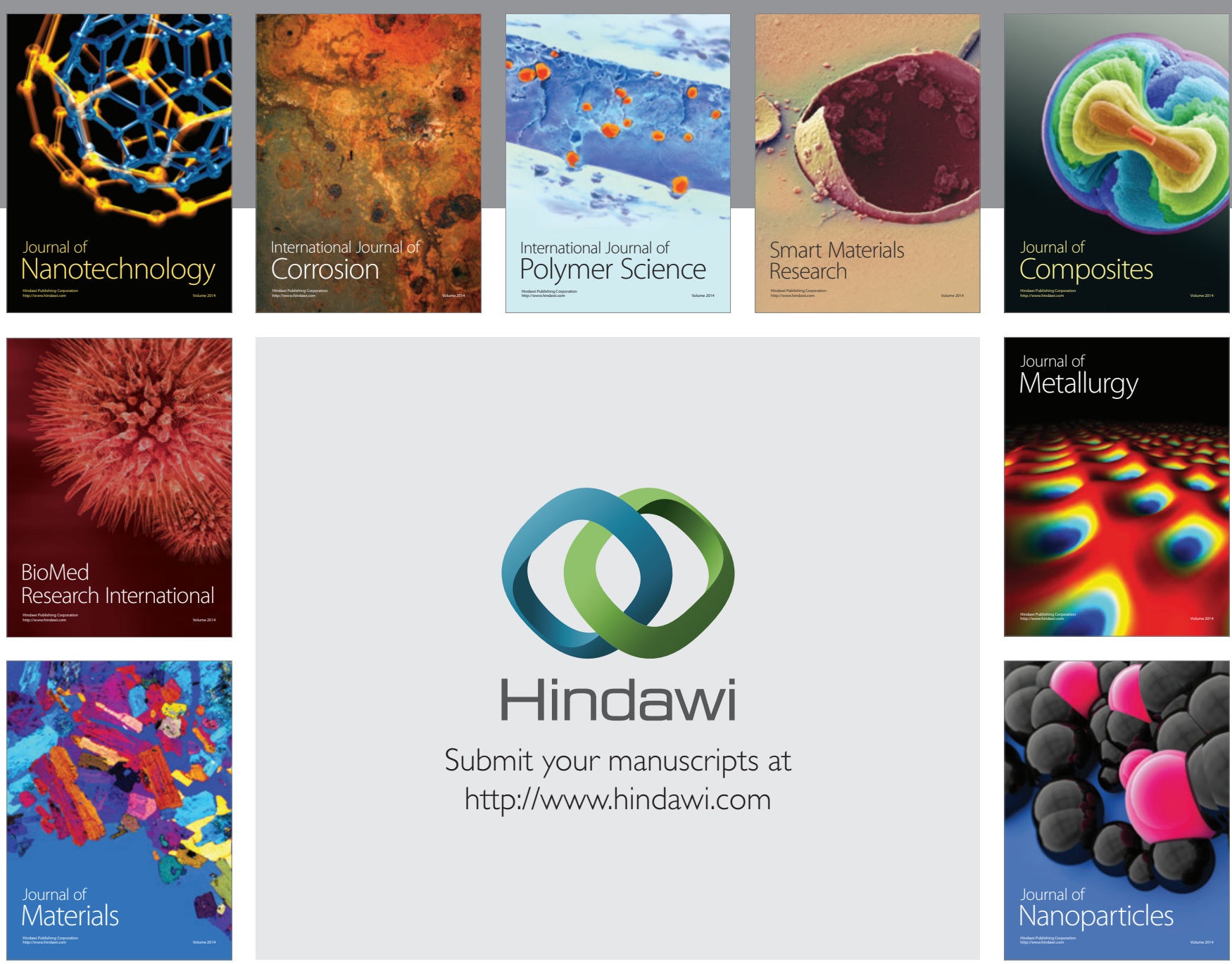

Submit your manuscripts at http://www.hindawi.com
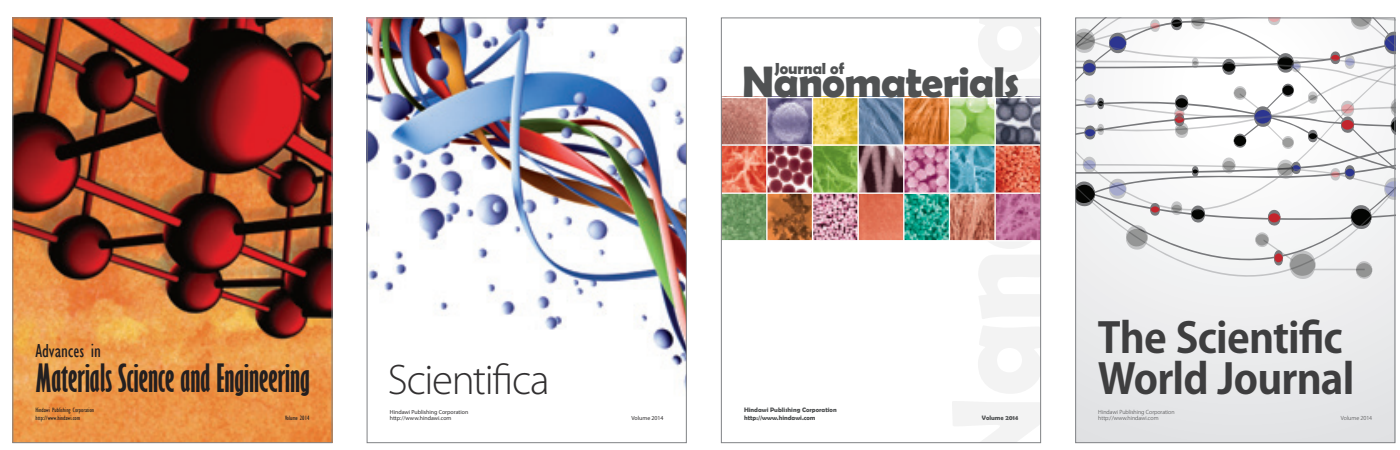

\section{The Scientific World Journal}
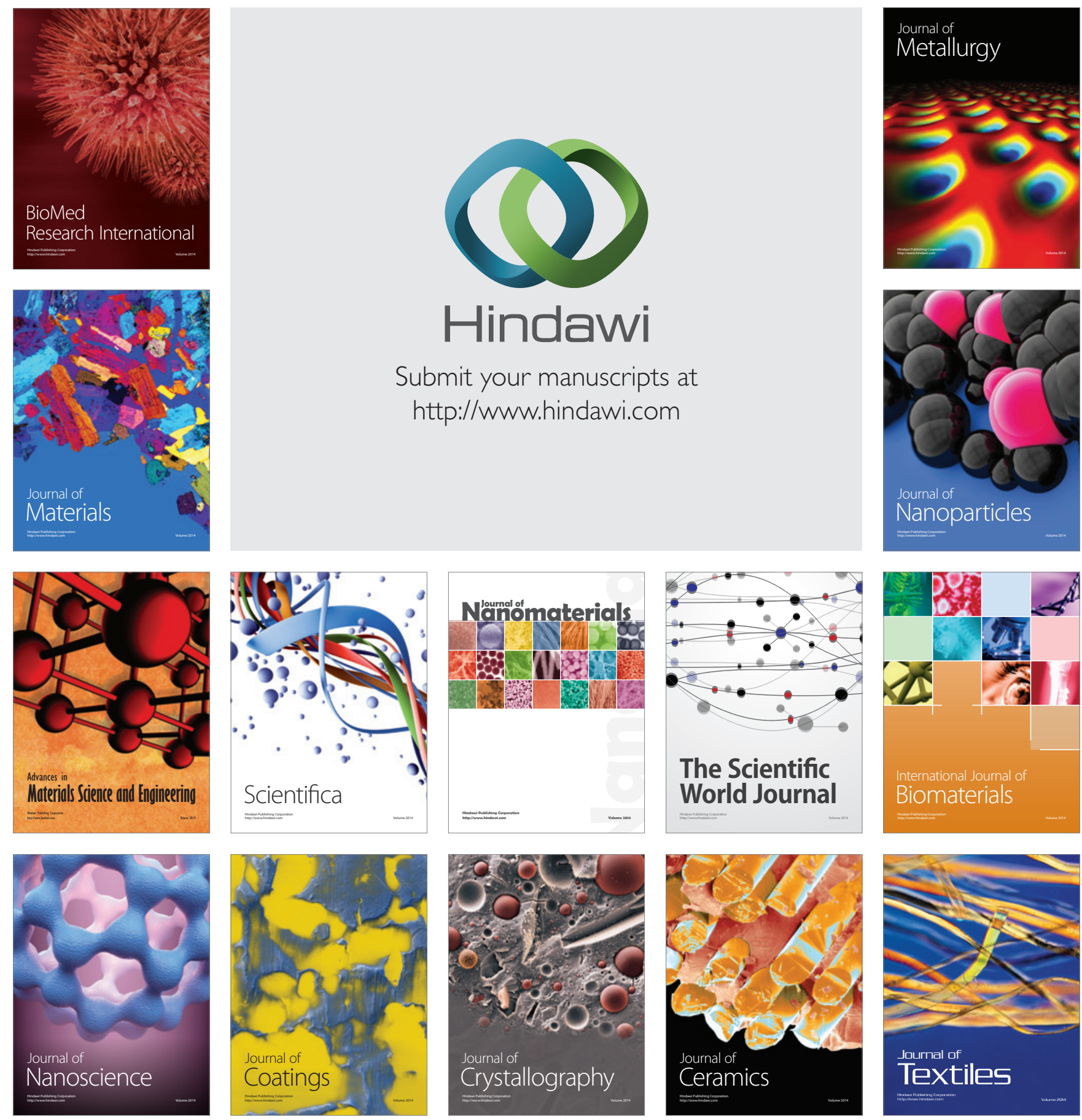